\title{
A MODEL FOR THE DYNAMIC ANALYSIS OF A BELT TRANSMISSION USING THE DAHL FRICTION MODEL
}

\author{
KRZYSZTOF KuBAS \\ University of Bielsko-Biala, Faculty of Mechanical Engineering and Computer Science, Bielsko-Biala, Poland \\ e-mail:kkubas@ath.bielsko.pl
}

\begin{abstract}
The paper presents a two-dimensional model of a belt transmission for dynamic analysis. It is assumed that the belt is modelled by links joined by spring-damping elements with its rotational and translational stiffness. Normal forces in the contact between the belt and the pulleys are implemented by assuming its stiffness and damping, whereas friction is modelled by the Dahl friction model. The calculation results are also presented in two specific cases of load of the belt transmission.
\end{abstract}

Keywords: belt transmission, dynamic analysis, Dahl friction model

\section{Introduction}

In the author's earlier papers, two-dimensional models of belt transmissions using the Threlfall friction model (Kubas, 2015) and a model including microslip were developed (Kubas, 2014). In the paper (Kubas, 2014), assumptions and requirements made during the process of model development were presented. In the present paper, the Dahl friction model (Dahl, 1968) is assumed as one in the group of dynamic friction models that allows one to include stiffness of contact areas.

Papers should be mentioned here in which friction was modelled between rubber and other materials. The most well known are works related to automotive engineering, especially those dealing with the modelling of friction between the tyre and the road, see e.g. often cited work by Canudas de Wit et al. (2003). Among the proposed friction models, also presented in the paper above was the Dahl friction model. The paper (Canudas de Wit et al., 2003) introduced another dynamic friction model - the LuGre model, which allows one to include the Stribeck effect.

Leamy and Wasfy $(2002 \mathrm{a}, \mathrm{b})$ presented belt transmission models with a piecewise linear friction model with the possibility of predicting belt creep. It is called the Coulomb-like trilinear creep-rate-dependent friction model. Another sample model was presented in (Kim et al., 2011) and was called the elastic/perfectly-plastic friction law (EPP).

A group of papers should also be mentioned in which the Dahl friction model is applied as a way of modelling friction in the revolute joint of a belt tensioner, see e.g. Bastien et al. (2007) and Chatlet et al. (2008).

The changing belt and chain transmissions research objectives over the centuries and more important works were presented by Fawcett (1981).

\section{Mathematical model}

The model presented in an earlier paper (Kubas, 2014) of a belt transmission was modified by changing it into a friction model. As presented in the above-mentioned work, it was assumed that the belt would be divided into $n_{b}$ bodies. Each neighbouring pair of bodies was joined by the SDE with proper translational and bending stiffness and damping parameters (Fig. 1). 


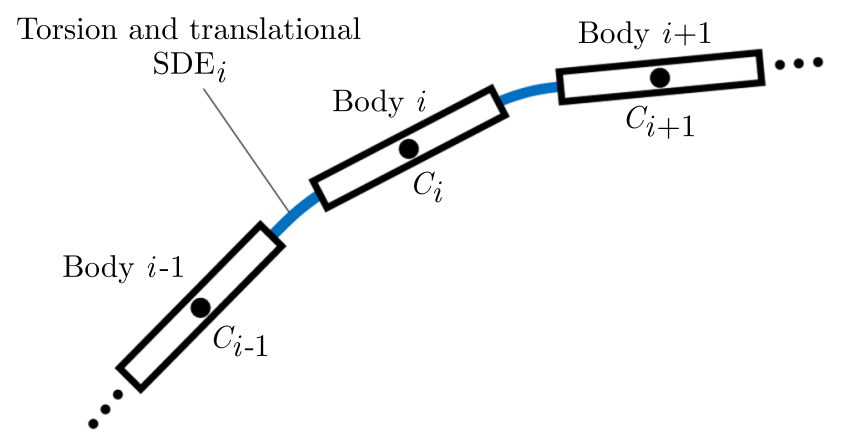

Fig. 1. Assumed belt model with translational and torsion SDEs

For each body $i\left(i=1, \ldots, n_{b}\right)$, it was assumed that there were three generalised coordinates (Fig. 2): translations $x_{i}$ and $y_{i}$ and rotation by an angle $\varphi_{i}$ relative to the mass centre. The generalised coordinates in the body $i$ are presented in Fig. 2 .

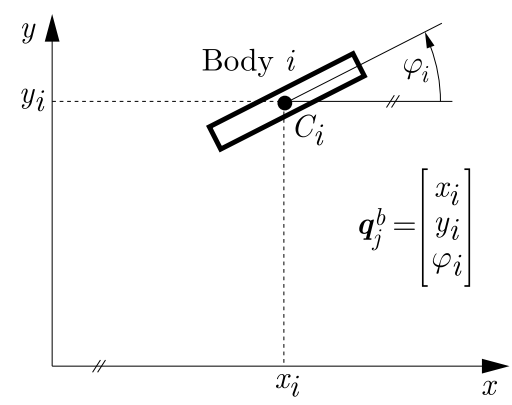

Fig. 2. Generalised coordinates of the belt body $i$

It was also assumed that there were $n_{p}$ pulleys in the transmission "lying" in the $x y$ plane and rotating around the axis parallel to $z$ with a rotation angle $\theta_{j}\left(j=1, \ldots, n_{p}\right)$. Therefore, the vector of the generalised coordinates takes the form

$$
\mathbf{q}^{\mathrm{T}}=\left[\mathbf{q}_{1}^{b^{\mathrm{T}}}, \ldots, \mathbf{q}_{i}^{b^{\mathrm{T}}}, \ldots, \mathbf{q}_{n_{b}}^{b}{ }^{\mathrm{T}}, \theta_{1}, \ldots, \theta_{j}, \ldots, \theta_{n_{p}}\right]
$$

where $\mathbf{q}_{i}^{b}$ is the vector of generalised coordinates of the body $i$ (presented in Fig. 2).

\subsection{Spring-damping elements}

The values of forces and torques in the translational and torsion SDE connecting the body $i-1$ with the body $i$ are described by the Kelvin-Voigt (Voigt, 1892) relations

$$
\begin{aligned}
& F_{i}^{L t r a}=F_{i-1}^{R t r a}=c_{t r a} \Delta l_{i}^{L}+b_{t r a} \Delta i_{i}^{L} \\
& M_{i}^{\text {Ltor }}=M_{i-1}^{\text {Rtor }}=c_{\text {tor }}\left(\varphi_{i}-\varphi_{i-1}\right)
\end{aligned}
$$

where $F_{i}^{L t r a}, F_{i}^{R t r a}$ are values of translational forces in the left SDE (connecting the body $i$ with the body $i-1$ ) and the right $\operatorname{SDE}$ (connecting the body $i$ with the body $i+1$ ), respectively; $M_{i}^{\text {Ltor }}, M_{i}^{\text {Rtor }}$ - bending torques in the left SDE and right SDE, respectively; $c_{t r a}, b_{t r a}$ - translational stiffness and damping coefficients in SDE; $c_{t o r}$ - torsional stiffness coefficient in SDE; $\Delta l_{i}^{L}, \Delta i_{i}^{L}$ - translational deformation and deformation speed of SDE.

\subsection{Model of the belt-pulley contact}

A vector notation of the forces is used to take into account the contact between the belt bodies and pulleys in the transmission. A diagram of the assumed distribution of forces acting on the belt body $i$ at the period of contact with the pulley $j$ is presented in Fig. 3. 


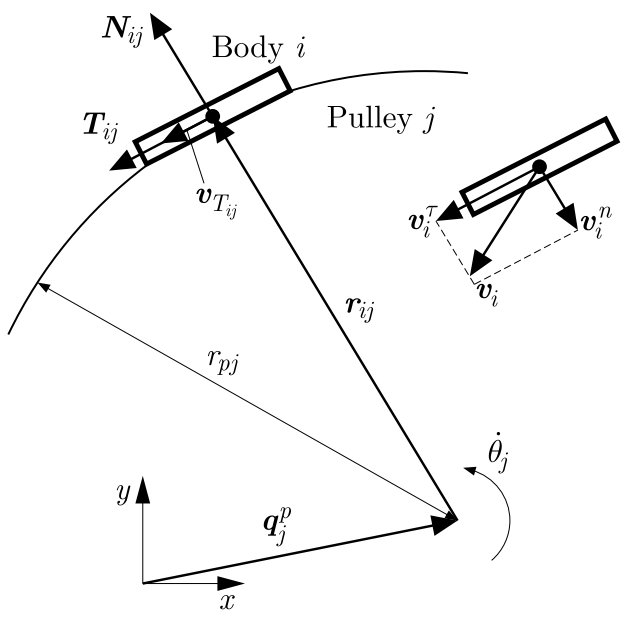

Fig. 3. Assumed configuration of the velocity components of the belt body $i$ and forces acting on this body from the pulley $j$

It is assumed that the force components, i.e. normal force $\mathbf{N}_{i j}$ and friction force $\mathbf{T}_{i j}$, would be applied to the mass centre of each body which has contact with one of the pulleys.

As is shown in Fig. 3, the vector $\mathbf{r}_{i j}$ is orientated from the centre of the pulley $j$ to the mass centre of the body $i$. If there is contact between the body and the pulley but the value of the normal force $\mathbf{N}_{i j}$ is still zero, the length of this vector equals an arbitrary value $r_{p j}$. Thus, at the time of a non-zero normal force, there is an inequality: $r_{i j}<r_{p j}$. The position of the pulley centre in the global coordinate system is specified via the vector $\mathbf{P}_{p j}$.

The vector $\mathbf{r}_{i j}$ can be determined from the following formula

$$
\mathbf{r}_{i j}=\mathbf{q}_{i}^{b}-\mathbf{q}_{j}^{p}
$$

A versor (unit vector) according to the direction and sense of the vector $\mathbf{r}_{i j}$ equals

$$
\overline{\mathbf{r}}_{i j}=\frac{\mathbf{r}_{i j}}{\left|\mathbf{r}_{i j}\right|}
$$

The penetration depth of the belt body $i$ with the pulley $j$ can be determined from the formula

$$
p_{i j}=r_{p j}-\left|\mathbf{r}_{i j}\right|
$$

Assuming that the linear velocity of the centre of the pulley is zero, then the value of penetration velocity $\dot{p}_{i j}$ is equal to the value of the normal velocity component $\mathbf{v}_{i}^{n}$ (Fig. 3). This value is determined on the basis of the following scalar product

$$
\dot{p}_{i j}=\left|\mathbf{v}_{i}^{n}\right|=-\mathbf{v}_{i}^{\mathrm{T}} \cdot \overline{\mathbf{r}}_{i j}
$$

where $\mathbf{v}_{i}$ is the velocity of the mass centre of the body $i$.

Since the contact force $\mathbf{N}_{i j}$ formed during contact between the body $i$ and the pulley $j$ has a consistent direction with the direction of the versor $\overline{\mathbf{r}}_{i j}$, then

$$
\mathbf{N}_{i j}=N_{i j} \overline{\mathbf{r}}_{i j}
$$

The value of this force is determined in a similar form as given in the paper (Cepon et al., 2010), in which the authors proved a nonlinear relation between the penetration depth and the normal force

$$
N_{i j}\left(p_{i j}, \dot{p}_{i j}\right)=c_{1} p_{i j}^{2}+c_{2} p_{i j}+b \dot{p}_{i j}
$$

where $c_{1}, c_{2}$ are belt-pulley contact stiffness coefficients, $b$ - belt-pulley contact damping coefficient. 


\subsection{Dahl friction model}

The value of the relative velocity $\mathbf{v}_{\text {Tij }}$ between the body $i$ and the pulley $j$ is needed to calculate the friction force (shown in Fig. 3). The way of calculating this velocity was presented in an earlier work (Kubas, 2014).

As was mentioned before, the Dahl model is assumed as presented in (Dahl, 1968)

$$
\mu_{i j}=\left\{\begin{array}{lll}
\left(\mu_{0 i j}-\mu_{k}\right) \exp \left(-\frac{\sigma}{\mu_{k}}\left(x_{i j}-x_{0 i j}\right)\right)+\mu_{k} & \text { for } & v_{\text {Tij }} \geqslant 0 \\
\left(\mu_{0 i j}+\mu_{k}\right) \exp \left(-\frac{\sigma}{\mu_{k}}\left(x_{i j}-x_{0 i j}\right)\right)-\mu_{k} & \text { for } & v_{T i j}<0
\end{array}\right.
$$

where $v_{\text {Tij }}$ is the relative velocity between two moving parts, $x_{0 i j}-$ starting value of displacement at the moment of change of sense of the relative velocity, $\mu_{0 i j}$ - starting value of the friction coefficient (according to $x_{0 i j}$ displacement), $x_{i j}$-displacement, $\mu_{k}$-dynamic friction coefficient, $\sigma-$ stiffness coefficient of the friction joint.

The shape of an exemplary displacement-dependent friction characteristic is presented in Fig. 4.

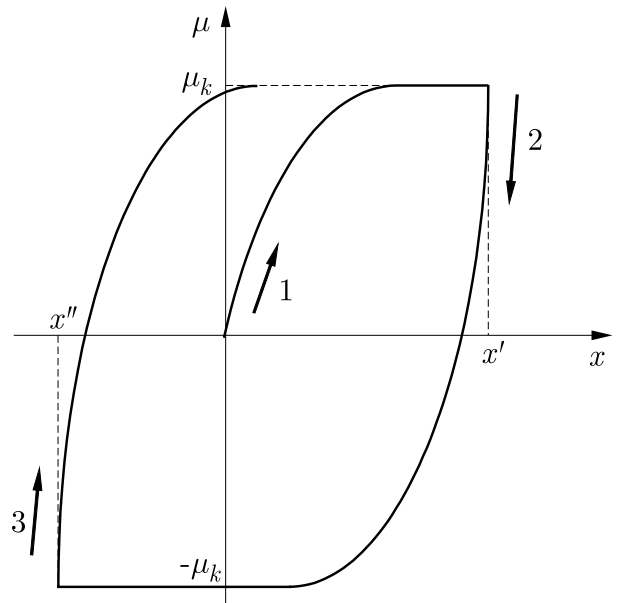

Fig. 4. Exemplary shape of a Dahl friction characteristic as a function of displacement

First, the starting value of displacement is $x_{0 i j}$ and the starting value of friction coefficient $\mu_{0 i j}$ is equal to 0 . When the two corresponding layers start to move and the relative velocity $\dot{x}$ is positive (in relation to the assumed local coordinate system), the friction coefficient

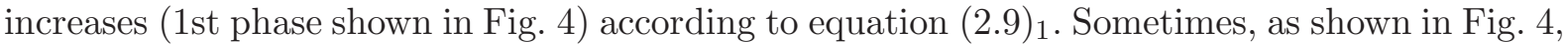
the relative velocity can reach the value of $\mu_{k}$ (especially when its direction does not change in a relatively long period of time). When the direction of the velocity changes to the opposite (negative values), the starting value of the displacement and the starting value of the friction coefficient should change to the values: $x_{0 i j}=x^{\prime}, \mu_{0 i j}=\mu_{k}$. The friction coefficient starts to decrease (2nd phase) according to equation $(2.9)_{2}$ and can reach an arbitry value of $-\mu_{k}$ (this situation is shown in Fig. 4). When the velocity changes again into positive values, the friction coefficient increases again (3rd phase), and $x_{0 i j}=x^{\prime \prime}, \mu_{0 i j}=-\mu_{k}$.

The value of the friction force can be, therefore, described as

$$
T_{i j}=\mu_{i j} N_{i j}
$$

The direction of the friction force vector is opposite to the relative velocity $\mathbf{v}_{\text {Tij }}$. 


\subsection{Equations of motion}

Equations of motion of the body $i$ take the form

$$
\begin{aligned}
& m_{i} \ddot{x}_{i}=\overline{\mathbf{X}}^{\mathrm{T}} \cdot\left[\mathbf{F}_{i}^{\text {Ltra }}+\mathbf{F}_{i}^{\text {Rtra }}+\sum_{j=0}^{n_{p}-1}\left(\mathbf{N}_{i j}+\mathbf{T}_{i j}\right)+m_{i} \mathbf{g}\right] \\
& m_{i} \ddot{y}_{i}=\overline{\mathbf{Y}}^{\mathrm{T}} \cdot\left[\mathbf{F}_{i}^{\text {Ltra }}+\mathbf{F}_{i}^{\text {Rtra }}+\sum_{j=0}^{n_{p}-1}\left(\mathbf{N}_{i j}+\mathbf{T}_{i j}\right)+m_{i} \mathbf{g}\right] \\
& I_{z_{i}} \ddot{\varphi}_{i}=\overline{\mathbf{Z}}^{\mathrm{T}} \cdot\left(\mathbf{M}_{i}^{\text {Ltra }}+\mathbf{M}_{i}^{\text {Rtra }}+\mathbf{M}_{i}^{\text {Ltor }}+\mathbf{M}_{i}^{\text {Rtor }}\right)
\end{aligned}
$$

where $\overline{\mathbf{X}}^{\mathrm{T}}=[1,0,0], \overline{\mathbf{Y}}^{\mathrm{T}}=[0,1,0], \overline{\mathbf{Z}}^{\mathrm{T}}=[0,0,1]$ - versors consistent with the axes $x, y, z$, respectively, of the global coordinate system, $\mathbf{M}_{i}^{\text {Ltra }}=-\mathbf{l}_{i}^{\prime} \times \mathbf{F}_{i}^{\text {Ltra }}, \mathbf{M}_{i}^{\text {Rtra }}=\mathbf{l}_{i}^{\prime} \times \mathbf{F}_{i}^{\text {Rtra }}$, $\mathrm{g}$ - vector of gravitational acceleration.

It is assumed that the pulleys motion will take place from the set torques. The equation of motion of the pulley $j$ has the form

$$
I_{z_{j}} \ddot{\theta}_{j}=M_{d j}-\sum_{i=0}^{n_{b}-1} \overline{\mathbf{Z}}^{\mathrm{T}} \cdot \mathbf{M}_{T i j}
$$

where $I_{z_{j}}$ is the mass moment of inertia of the pulley $j, M_{d j}$ - the set torque, $\mathbf{M}_{T i j}=\overline{\mathbf{r}}_{i j} \times \mathbf{T}_{i j}$ - friction torque acting from the belt body $i$.

The resistance torque acting on the pulley can be included in the assumed model. A negative value of $M_{d j}$ (e.g. constant or dependent on velocity $\dot{\theta}_{j}$ ) should be taken in this case.

Case 1: Analysis with a driving torque of $100 \mathrm{Nm}$

A two-pulley belt transmission with the same radius $r_{p}=0.1 \mathrm{~m}$ is taken into analysis. The scheme of the assumed transmission is presented in Fig. 5. The distance between the pulleys is $l_{p}=0.290 \mathrm{~m}$. The analysed belt is $1.2 \mathrm{~m}$ in length. The friction coefficient, translational and rotational stiffness and damping coefficients are assumed based on own related works and (Čepon and Boltežar, 2009; Čepon et al., 2009, 2010). The number of belt elements is assumed as $n_{b}=60$ and the coefficient as $\sigma=10000 \mathrm{~m}^{-} 1$. One belt element and the neighbouring spring-damping element (on the left side of the belt element) are chosen to show some example calculation results. The selected belt element is also presented in Fig. 5 as case 1.

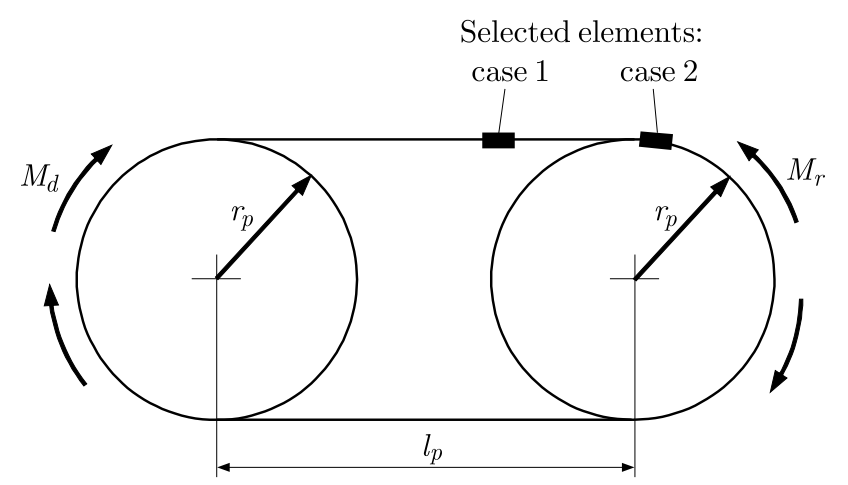

Fig. 5. Assumed belt transmission with a selected belt element

The torque applied in the driving pulley is also assumed as a partially-linear time-depended function. From 0 to $0.3 \mathrm{~s}$, the value of the torque decreased from 0 to $-50 \mathrm{Nm}$. After that moment, it remained constant. 
The resistance torque applied in the driven pulley is described as a function of its angular velocity

$$
M_{d 2}=\frac{100}{2 \pi} \dot{\theta}_{2}
$$

All of the presented calculation results omit the stabilisation phase of the analysed model.

The calculated driving and resistance torques are presented in Fig. 6.

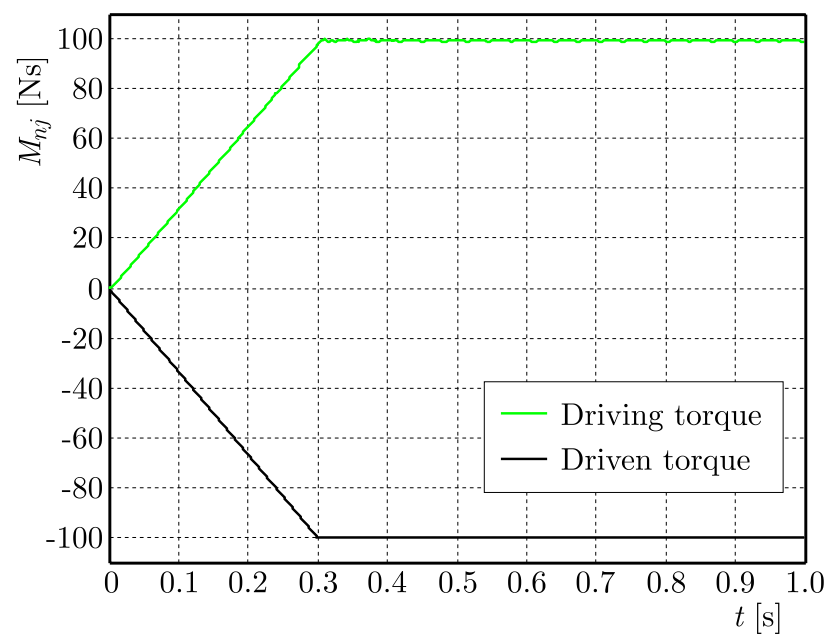

Fig. 6. Driving and driven torques applied to the pulleys in case 1

The achieved angular velocities of the driving and driven pulleys are presented in Fig. 7. As can be observed, after $0.3 \mathrm{~s}$ the angular velocities remain constant with a value almost equal to $2 \pi \mathrm{rad} / \mathrm{s}$. Some disturbances can also be observed as a result of the assumed discrete model of the belt. It is worth mentioning that this effect can be reduced even more by assuming more belt elements; this will of course cause an increase in the number of equations of motion.

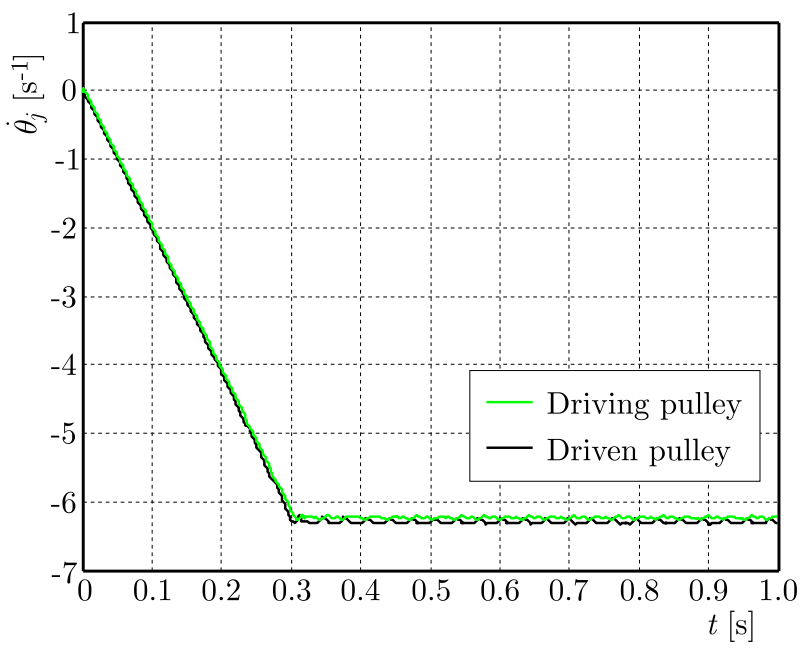

Fig. 7. Calculated values of angular velocities of the pulleys

In the next two figures (Figs. 8a and 8b), the longitudinal deformation and resulting force in a chosen spring-damping element is presented. As has been mentioned, it is located next to the chosen rigid element shown in Fig. 5. As can be observed, this element is located in the passive part of the belt at the moment that the transmission starts. The values of deformation and force decrease. Some disturbances and an increase in the values can be observed just over 
$0.3 \mathrm{~s}$. From this moment, the rigid element is located on the driven pulley. This situation lasts to about $0.82 \mathrm{~s}$. After this time period, it moves in the active part of the belt.

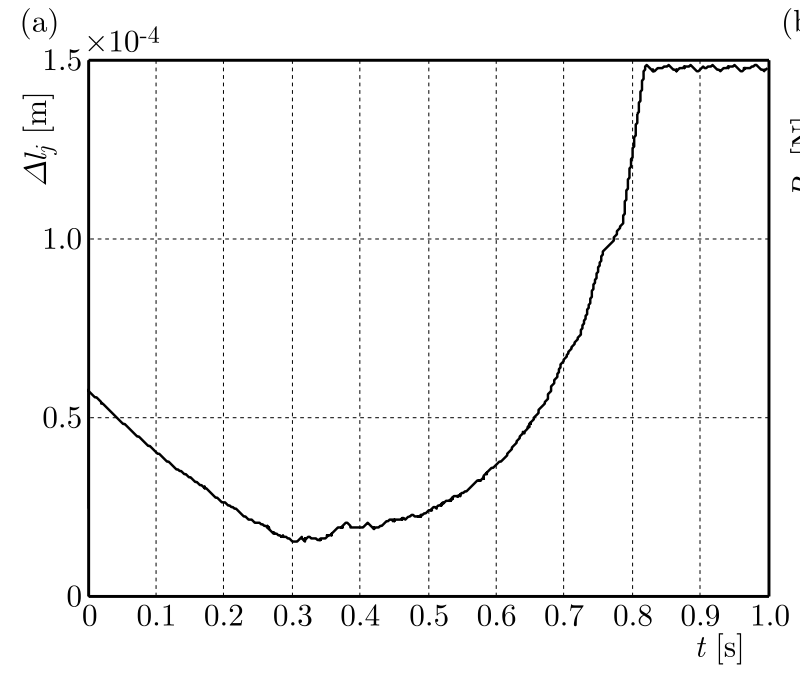

(b)

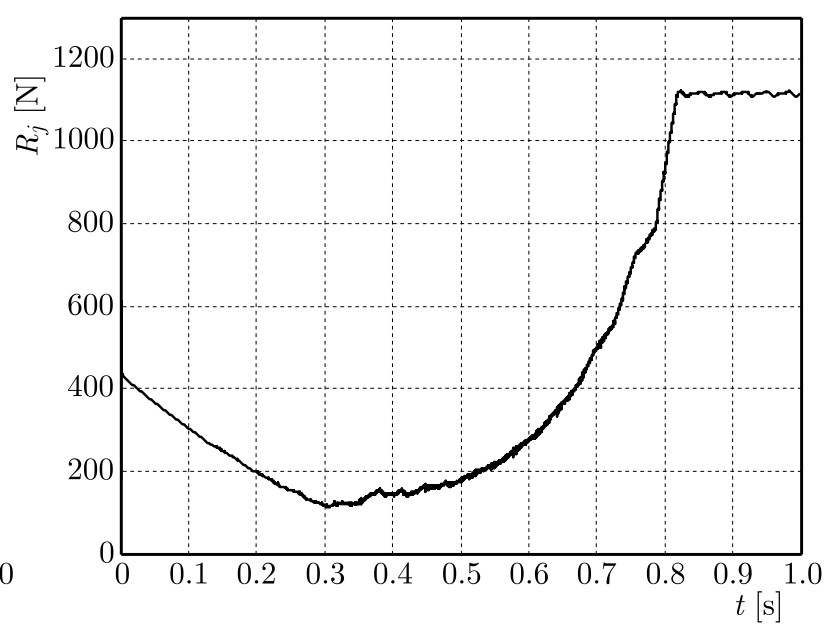

Fig. 8. Longitudinal deformation and reaction force in the selected SDE: (a) longitudinal deformation, (b) reaction force

As can be observed from the shapes in the figures, the reaction force in the spring-damping element mainly depends on the deformation. The dependence on the deformation velocity is negligible.

Figure 9 shows the reaction forces on each element at a chosen time of $0.4 \mathrm{~s}$. Elements with numbers 1-9 and 53-60 are located in the passive top part of the belt. The values of the force in this part are about $120 \mathrm{~N}$. Elements 23-39 are in the active bottom part of the belt. The values of the force in this part are about 1120 N. Elements located in the driven pulley correspond to numbers 10-22 (growing force), whereas those located on the driving pulley correspond to numbers 40-52 (decreasing force).

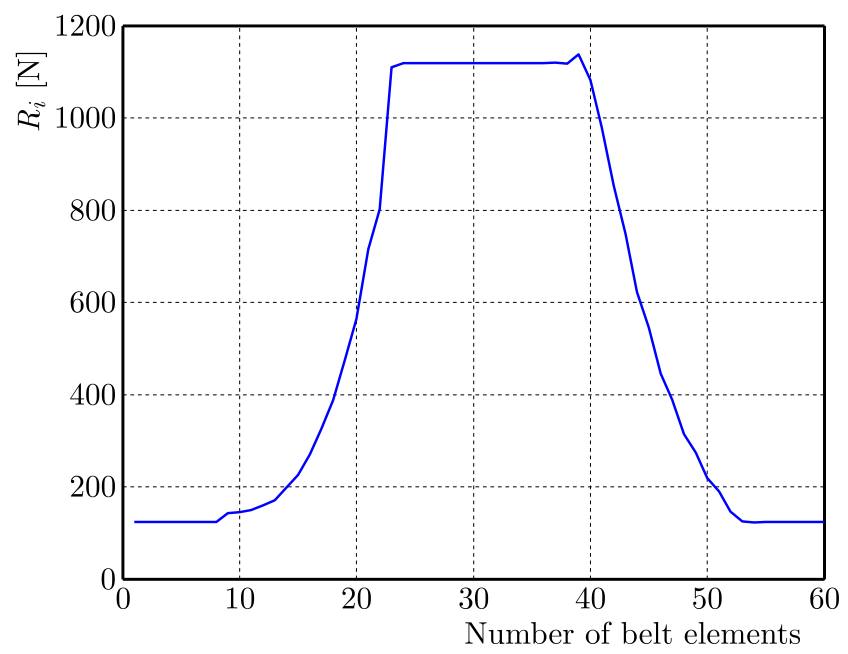

Fig. 9. Reaction forces in all SDEs at a selected time

In Figs. 10a and 10b, the calculated values of normal and friction forces acting on a selected rigid element from the driven pulley are presented. As can be observed, up to about $0.6 \mathrm{~s}$, the friction forces are smaller than the normal forces and do not correspond to the developed friction or slip (which is consistent with the results of the angular velocities shown in Fig. 7). From about $0.6 \mathrm{~s}$, the values start to be equal or almost equal. 
(a)

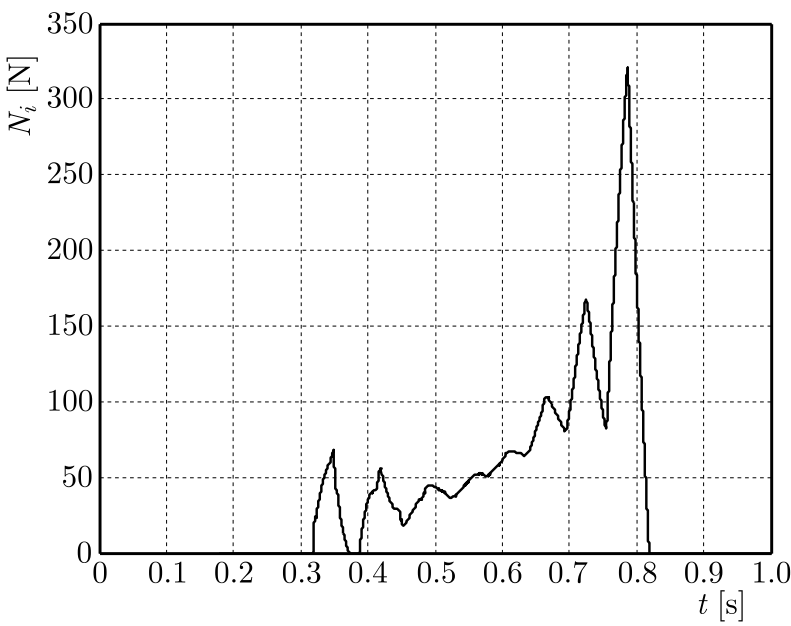

(b)

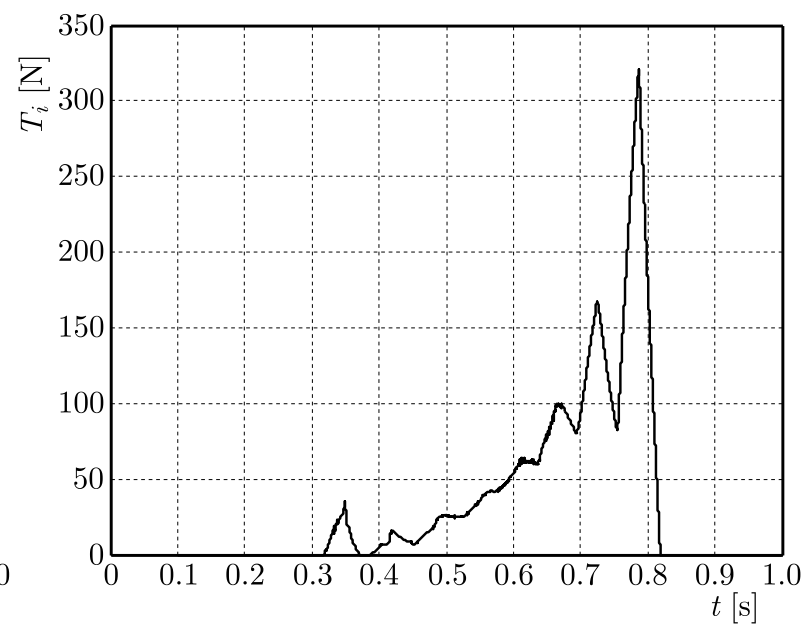

Fig. 10. Normal and friction forces acting on a selected belt element from the driven pulley:

(a) normal force, (b) friction force

In Fig. 11a, the calculated values of the friction coefficient of the selected belt element are shown. The values are negative because of the opposite direction of the friction force to the assumed positive direction of the pulley rotation (Fig. 3). As can be observed, when contact between the element and the driven pulley starts, the values of the friction coefficient increase rapidly to a value of about 0.59 and return to zero (from about $0.31 \mathrm{~s}$ to $0.38 \mathrm{~s}$ ). At this moment, the element loses contact with the pulley (this can also be observed in Fig. 10a). At about $0.39 \mathrm{~s}$, the belt element returns to the area of the pulley. Up to about $0.68 \mathrm{~s}$, the values increase to the assumed value $\mu_{k}$. The values remain constant from this time to about $0.82 \mathrm{~s}$. At about $0.82 \mathrm{~s}$, the element loses contact with the driven pulley and starts moving in the active part of the belt.

(a)

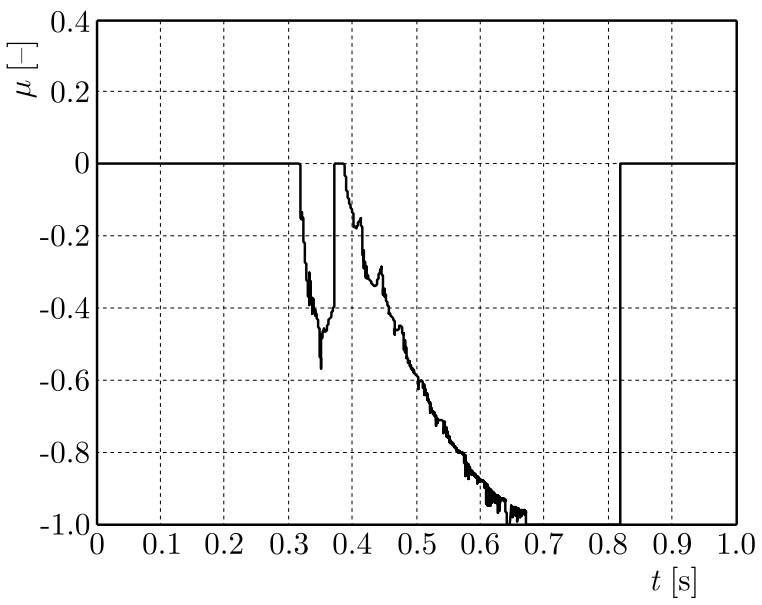

(b)

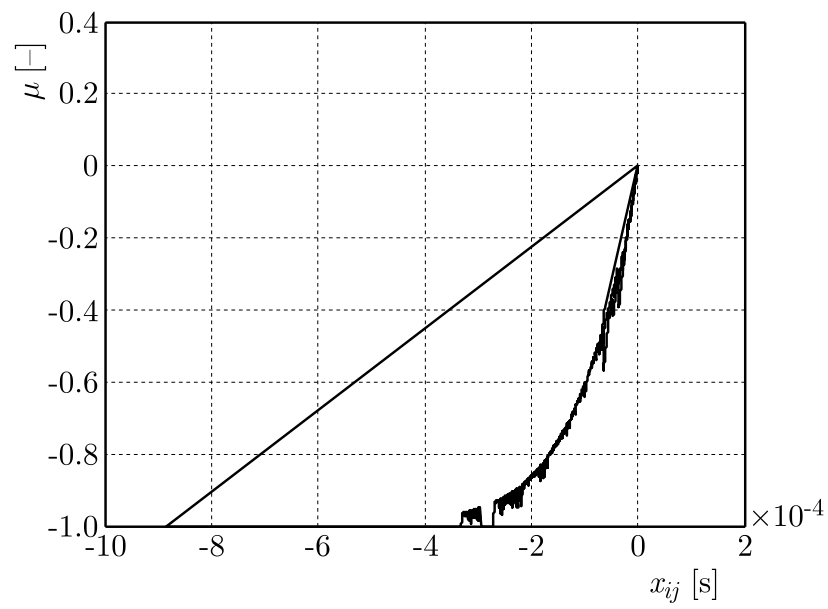

Fig. 11. Change of the friction coefficient: (a) in a selected belt element, (b) presented as a function of displacement

In Fig. 11b, the calculated values of the friction coefficient are shown as a function of displacement. As can be observed, the values asymptotically grow to the assumed value $\mu_{k}$. Two moments of changing the values to zero, as identified earlier, can be observed.

The disturbances of the changing values of the friction coefficient result from the changing direction of the relative velocity, which is presented in Fig. 12. 


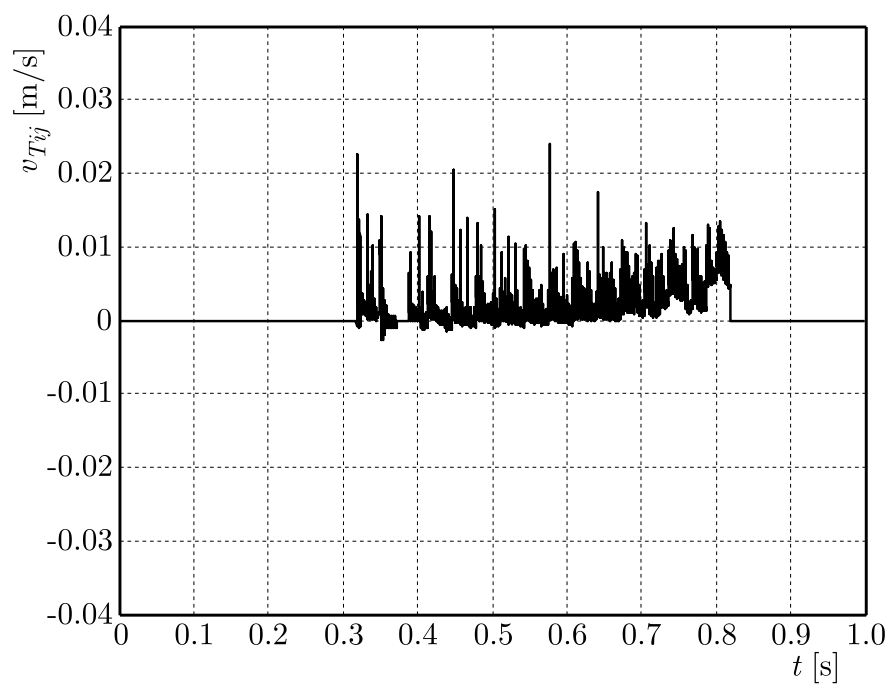

Fig. 12. Calculated relative velocity between a chosen rigid element and the driven pulley

The presented case of load causes a relatively large acceleration of the transmission. After velocity stabilisation, only friction in one direction can be analysed. Calculations with another drive and resistance torques are repeated.

Case 2: Oscillating torque

Next, the torque applied to the driving pulley as shown in Fig. 13 is assumed. As can be observed, first the torque is linear-changing from 0 (at the start) to $-50 \mathrm{Nm}$ (at time $0.25 \mathrm{~s}$ ). Next, the values increase to $50 \mathrm{Nm}$ (at $0.75 \mathrm{~s}$ ). At the end of analysis, the torque decreases to 0 . Simultaneously, the resistance torque of the passive pulley has opposite values.

This kind of torque loads the transmission in two opposite directions with a relatively small movement. The belt is slightly rotated clockwise. The chosen belt element (whose initial position is shown in Fig. 5 as case 2) moves toward the right side of the driven pulley.

The rest of the conditions (initial preload of the belt, dimensions of the transmission, etc.) are the same.

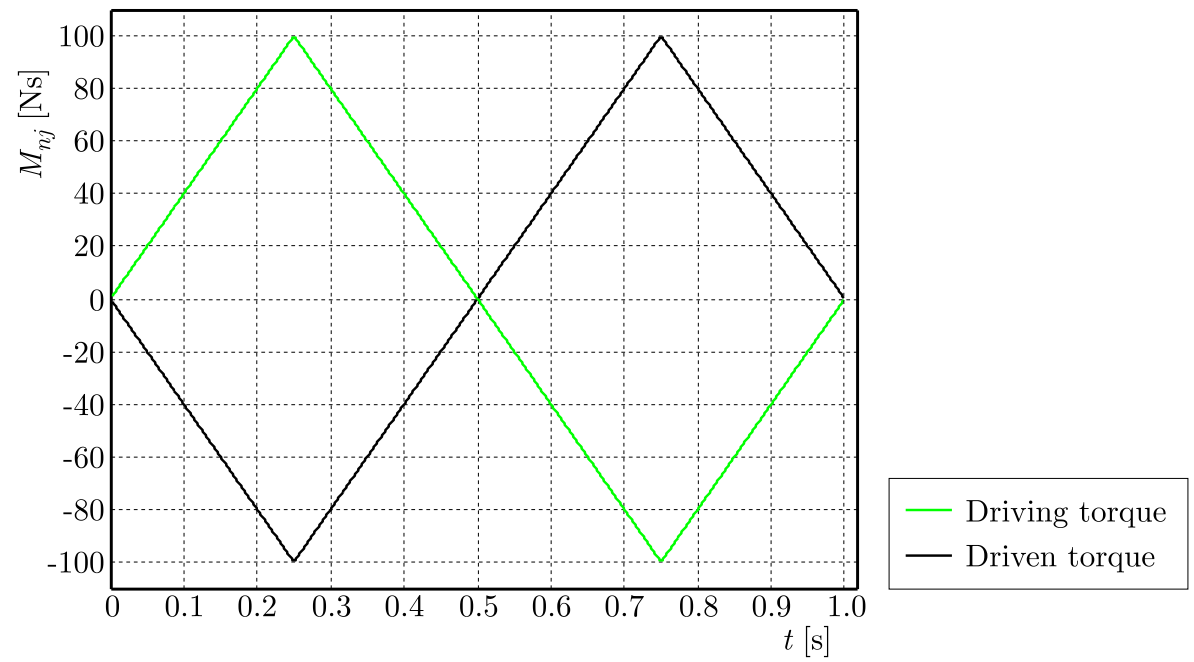

Fig. 13. Driving and driven torques applied to the pulleys in case 2

As shown in Fig. 14, the angular velocities of the driving and driven pulleys differ significantly. It can also be observed that the transmission oscillates in moments of the changing direction of 
these velocities (at time $0.25 \mathrm{~s}$ and $0.75 \mathrm{~s}$ ). Just before the oscillations, the velocities differ about $0.1 \mathrm{rad} / \mathrm{s}$.

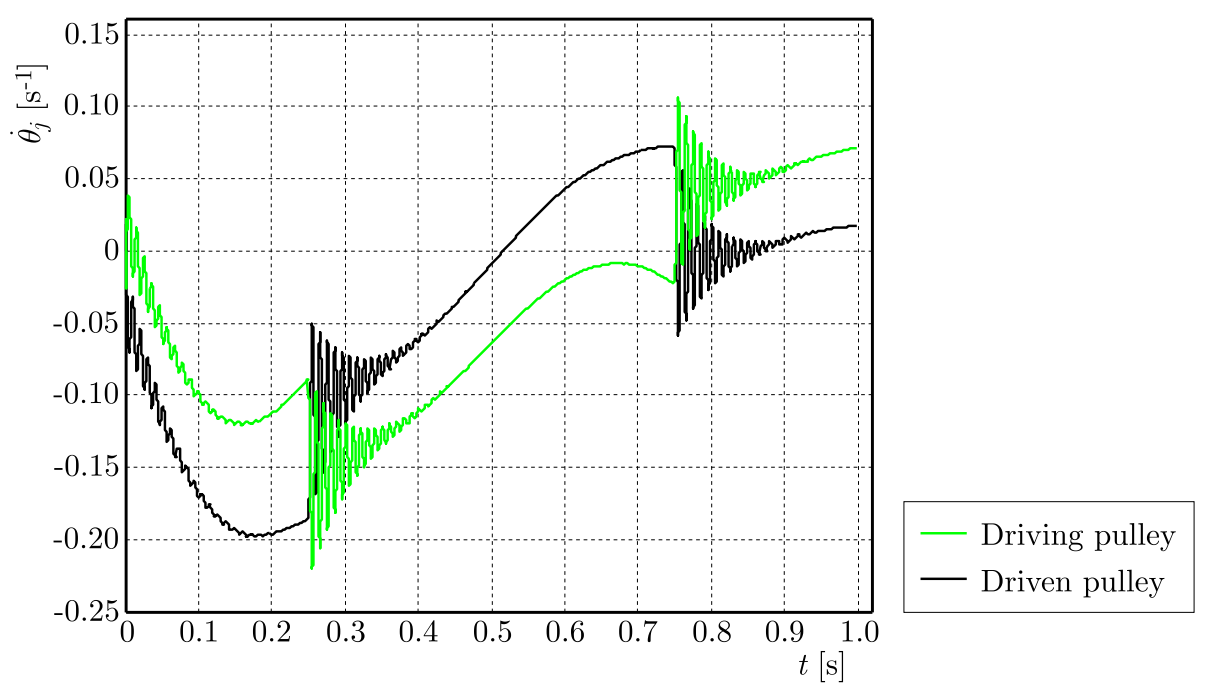

Fig. 14. Calculated angular velocities of the pulleys in case 2

Figure 15 shows the resulting reaction force in the selected spring-damping element. At the beginning, the force is about $420 \mathrm{~N}$ and decreases to about $100 \mathrm{~N}$. After changing the direction of the load torques, the force increases to about $1110 \mathrm{~N}$. At the end of the simulation the force achieves a value of about $580 \mathrm{~N}$.

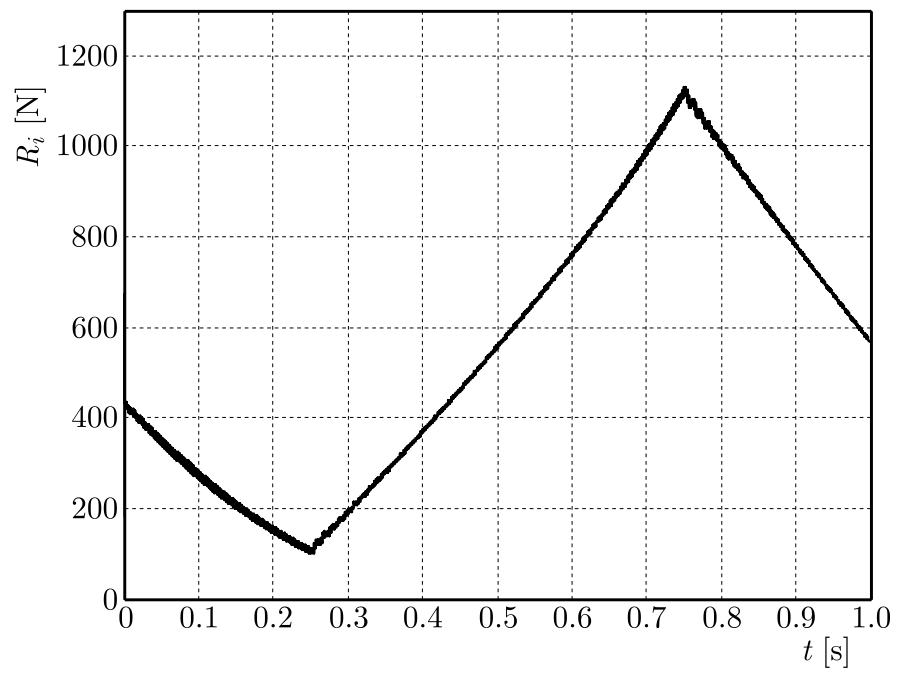

Fig. 15. Calculated values of the reaction force in a selected spring-damping element in case 2

Figure 16 shows the calculated normal and friction forces between the chosen belt element and the pulley. Because the selected belt element moves slowly to the right side of the driven pulley, where the normal force is larger, the absolute friction force also increases to about $190 \mathrm{~N}$.

After $0.75 \mathrm{~s}$, the selected belt element moves back and the absolute values of normal and friction forces start to decrease.

Figure 17a shows the calculated values of the friction coefficient. In this case, the friction between the selected belt element and the pulley achieves values of $-\mu_{k}$ and $\mu_{k}$, which means that in particular moments it is fully developed. It corresponds to intervals $0.16 \mathrm{~s}-0.25 \mathrm{~s}$ and $0.66 \mathrm{~s}-0.76 \mathrm{~s}$, respectively. 
(a)

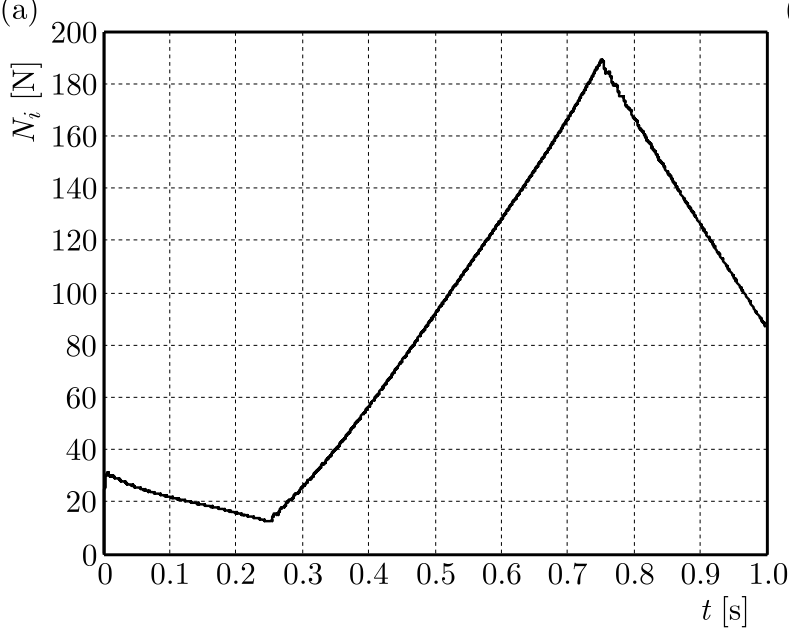

(b)

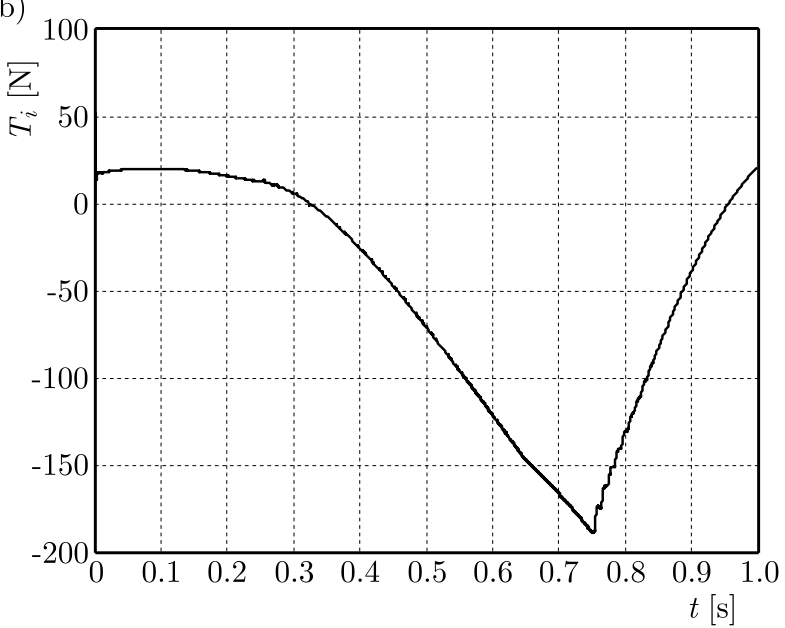

Fig. 16. Normal and friction forces of a selected belt element in case 2

(a)

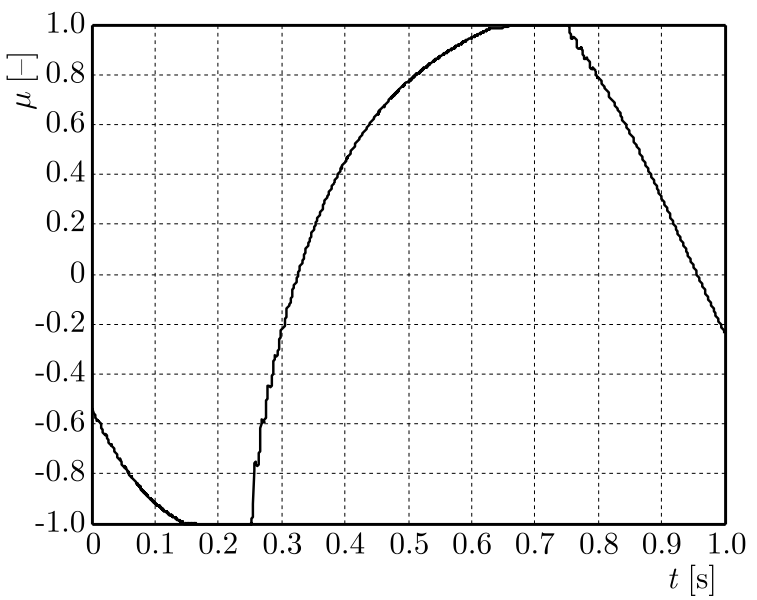

(b)

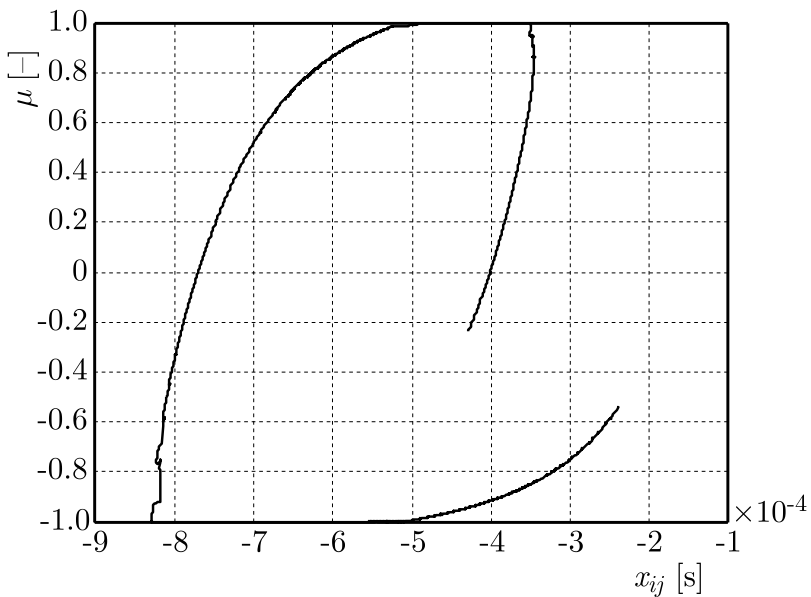

Fig. 17. Change of the friction coefficient: (a) in a selected belt element in case 2, (b) presented as a function of displacement in case 2

Figure 17b shows values of the friction coefficient as a function of displacement acting on a chosen element from the pulley. As can be observed, at the beginning the friction coefficient decreases to an arbitrary value $-\mu_{k}$ and the displacement is achieved at about $0.83 \mathrm{~mm}$. Then the direction of the relative velocity changes. After this moment, the values increase to a positive value of $\mu_{k}$. The relative displacement achieves in this phase about $0.45 \mathrm{~mm}$ (absolute displacement is about $0.38 \mathrm{~mm}$ ).

It has been decided to check how much the change in the $\sigma$ coefficient affected the calculation results. Figure 18 shows the friction coefficient courses as a function of the displacement in two cases: $\sigma=10000$ and $\sigma=5000$ as assumed earlier. As can be observed, the slope of the curve has changed.

Figure 19 shows the calculated angular velocities of the pulleys. A comparison of these courses with the courses from Fig. 14 shows slightly larger amplitudes of the vibrations. Because of the assumed smaller value of $\sigma$, the system became 'more flexible'. The contact and friction parameters will be investigated on a research stand in the future. 


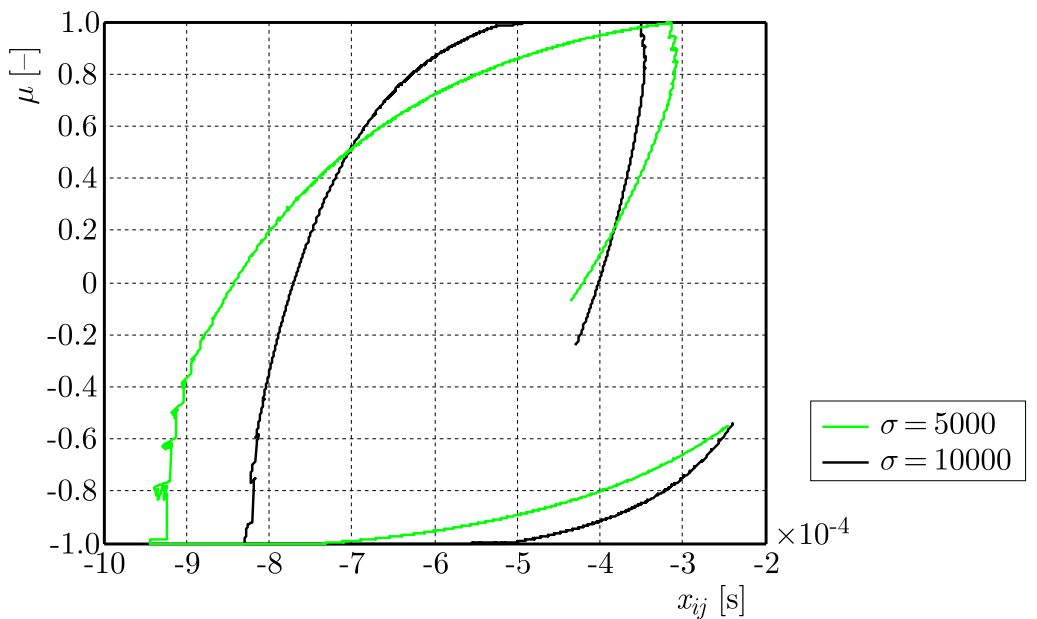

Fig. 18. Change of the friction coefficient presented as a function of displacement in case 2, for two different values of the coefficient $\sigma$

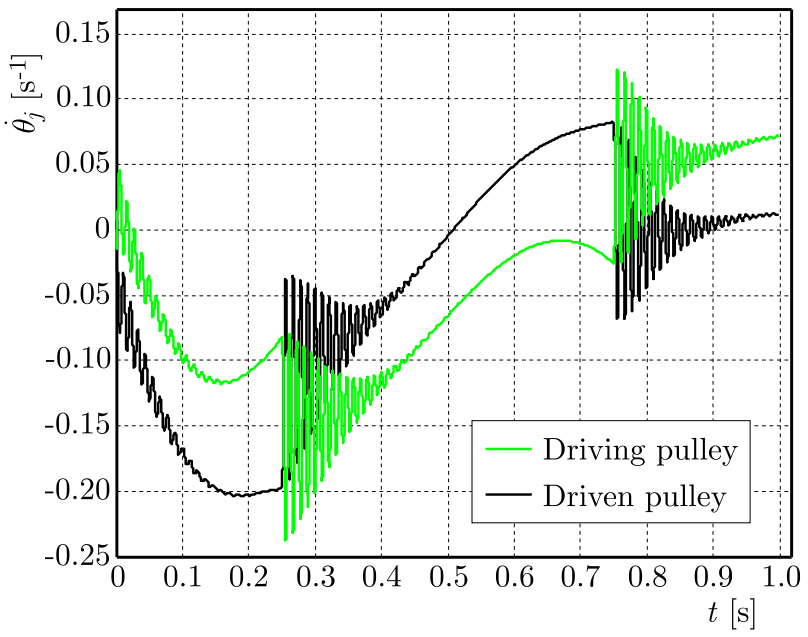

Fig. 19. Calculated angular velocities of pulleys in case 2 with the assumed coefficient $\sigma=5000$

\section{Conclusions}

As has been mentioned before, the proposed Dahl model does not include the Stribeck effect. This assumption is possible because the static and dynamic friction coefficients are approximately the same. It would be necessary to assume another friction model if they were different. The LuGre friction model is especially interesting and very popular (Canudas de Wit et al., 1995). In future works, it will be the main subject of investigation in belt transmission applications. It is also important to compare the prepared models with early mentioned models, especially with the EPP friction model, which also includes elasticity of the joint.

Of course, the most important is to compare them with real measurements made on a research stand. As mentioned before, a series of experiments on a specially built research stand is planned. The main subject of these experiments will be to measure the friction parameters between the belt and the pulley. The measurements will be done for a clean belt and with some impurities (oil, water, etc.). It has been noticed from first experiments made on a clean belt that friction between the belt and the pulley cannot be described with a relatively simple Euler formula. Its dependence on the belt preload or wrap angle can be more complicated. The friction can also depend on the rest time between movements of the transmission or belt slip, on the relative velocity between the belt and the pulley surfaces or even on the acceleration. 


\section{References}

1. Bastien J., Michon G., Manin L., Dufour R., 2007, An analysis of the modified Dahl and Masing models: Application to a belt tensioner, Journal of Sound and Vibration, 302, 4/5, 841-864

2. Canudas de Wit C., Olsson H., Astrom K.J., Lischinsky P., 1995, A new model for control of systems with friction, IEEE Transactions on Automatic Control, 40, 3

3. Canudas de Wit C., Tsiotras P., Velenis E., Basset M., Gissinger G., 2003, Dynamic friction models for road/tire longitudinal interaction, Vehicle System Dynamics, 39, 3, 189-226

4. Chatlet E., Michon G., Manin L., Jacquet G., 2008, Stick/slip phenomena in dynamics: Choice of contact model, Mechanism and Machine Theory, 43, 10, 1211-1224

5. Čepon G., BoltežAr M., 2009, Dynamics of a belt-drive system using a linear complementarity problem for the belt-pulley contact description, Journal of Sound and Vibration, 319, 1019-1035

6. Čepon G., Manin L., Boltežar M., 2009, Introduction of damping into the flexible multibody belt-drive model: A numerical and experimental investigation, Journal of Sound and Vibration, 324, 283-296

7. Čepon G., Manin L., BoltežAr M., 2010, Experimental identification of the contact parameters between a V-ribbed belt and a pulley, Mechanism and Machine Theory, 45, 1424-1433

8. Dahl P.R., 1968, A Solid Friction Model, Report No. TOR-0158(3107-18)-1, Aerospace Corporation Report

9. Fawcett J.N., 1981, Chain and belt drives - a review, Shock Vibrations Digest, 13, 5, 5-12

10. Kim D., Leamy M.J., Ferri A.A., 2011, Dynamic modeling and stability analysis of flat belt drives using an elastic/perfectly plastic friction law, ASME Journal of Dynamic Systems, Measurement, and Control, 133, 1-10

11. KubAs K., 2014, A two-dimensional discrete model for dynamic analysis of belt transmission with dry friction, The Archive of Mechanical Engineering, 61, 4, 571-593

12. Kubas K., 2015, A model for analysing the dynamics of a belt transmissions with a 5pk belt, The Archives of Automotive Engineering, 16, 1

13. LEAmy M.J., WASFy T.M., 2002, Analysis of belt-drive mechanics using a creep-rate-dependent friction law, Journal of Applied Mechanics, Transaction of ASME, 69, 6, 763-771

14. Leamy M.J., Wasfy T.M., 2002, Transient and steady-state dynamic finite element modeling of belt-drives, ASME Journal of Dynamic Systems, Measurement, and Control, 124, 4, 575-581

15. Voigt W., 1892, Ueber innere Reibung fester Körper, insbesondere der Metalle, Annalen der Phisik, 283, 671-693 